\title{
Characterization of Fractured Reservoirs Using a Combination of Downhole Pressure and Self-Potential Transient Data
}

\author{
Yuji Nishi and Tsuneo Ishido \\ GSJ, National Institute of Advanced Industrial Science and Technology (AIST), Tsukuba Central 7, Tsukuba, Ibaraki 305-8567, Japan \\ Correspondence should be addressed to Tsuneo Ishido, ishido-t@aist.go.jp
}

Received 1 July 2011; Revised 11 October 2011; Accepted 8 November 2011

Academic Editor: Laurence Jouniaux

Copyright ( $\odot 2012$ Y. Nishi and T. Ishido. This is an open access article distributed under the Creative Commons Attribution License, which permits unrestricted use, distribution, and reproduction in any medium, provided the original work is properly cited.

In order to appraise the utility of self-potential (SP) measurements to characterize fractured reservoirs, we carried out continuous $\mathrm{SP}$ monitoring using multi $\mathrm{Ag}-\mathrm{AgCl}$ electrodes installed within two open holes at the Kamaishi Mine, Japan. The observed ratio of SP change to pressure change associated with fluid flow showed different behaviors between intact host rock and fractured rock regions. Characteristic behavior peculiar to fractured reservoirs, which is predicted from numerical simulations of electrokinetic phenomena in MINC (multiple interacting continua) double-porosity media, was observed near the fractures. Semilog plots of the ratio of SP change to pressure change observed in one of the two wells show obvious transition from intermediate time increasing to late time stable trends, which indicate that the time required for pressure equilibration between the fracture and matrix regions is about 800 seconds. Fracture spacing was estimated to be a few meters assuming several micro-darcies $\left(10^{-18} \mathrm{~m}^{2}\right)$ of the matrix region permeability, which is consistent with geological and hydrological observations.

\section{Introduction}

Geothermal reservoirs are frequently found in fractured rock formations which are otherwise nearly impermeable. The fractures serve as conduits for the geothermal fluids, and the relatively low-permeability country rock provides the reservoir storage capacity. The spacing between the discontinuities (faults/fractures/joints) is obviously an important parameter in any mathematical description of fluid flow through fissured rocks. Apart from major fault zones which is necessary to be modeled individually, the spacing $(\lambda)$ of the discontinuities is usually small compared to the reservoir dimensions $(L)$. Hence, the fissured rock mass can be treated as a continuum on an intermediate length scale $(l)$, with $\lambda<l<L[1]$.

One continuum description of such a system is provided by the so-called "double-porosity model" (e.g., [2, 3]), in which the fractures and the porous matrix blocks are regarded as two separate but overlapping continua. But this approach is of limited utility in geothermal reservoir engineering mainly due to employment of analytical approximations for mass and energy exchange between the two continua. Pruess and Narasimhan [4] developed the "MINC" (multiple interacting continua) model, which circumvents the difficulties associated with the estimation of mass and energy exchange between the fracture and matrix regions. The "MINC" method was successfully applied to explain, for example, "excess enthalpy" phenomena [5]; when a well is drilled into the fractured reservoir and fluid is withdrawn, the enthalpy of the stable fluid discharge is often anomalously high-sometimes the well discharges steam alone even though the reservoir is initially filled with almost liquid water. Pritchett and Garg [1] showed that two time constants are key parameters in characterizing twophase flow in fractured reservoirs: the time required for pressure equilibration $\left(\tau_{\text {pe }}\right)$ and temperature equilibration $\left(\tau_{\mathrm{hc}}\right)$ between the fracture and matrix regions, both of which are proportional to the square of fracture spacing $\left(\lambda^{2}\right)$.

In the problem of cold water injection into a geothermal reservoir, the cold water advancement in a fractured reservoir that is represented as "MINC" double-porosity medium will be very different from that in a reservoir that can be represented by equivalent porous medium. In the fractured 
reservoir, the cold water will advance along the fracture zones, gradually extract heat from the adjacent rock matrix, and eventually arrive at the production wells. If the representative time scale $t_{R}$ is shorter than $\tau_{\mathrm{hc}}$, the cold water has not fully heated up by then, which brings about undesired effects on heat recovery from decreasing fluid enthalpies. To achieve more complete heat recovery from the matrix rocks, we need to reduce the injection rate so as that the representative time scale is sufficiently longer than $\tau_{\text {hc }} \propto \lambda^{2}$ (e.g., $\left.[6,7]\right)$.

It is highly desirable to know the time constants $\tau_{\mathrm{pe}}$ and $\tau_{\mathrm{hc}}$ in advance for prediction of two-phase flow behavior and/or cold water advancement in fractured geothermal reservoirs. Ishido and Pritchett [8] extended the so-called EKP-postprocessor [9] to apply it to fractured reservoirs represented by MINC media. They carried out pressure-transient simulations and calculated associated "self-potential transients" by using the extended EKPpostprocessor and showed that much more pronounced differences will be brought about in the self-potential transients between competing "fractured/MINC" and "porousmedium" descriptions of the same reservoir than is the case for pressure transients. They suggested that combining continuous pressure and SP measurements may therefore provide a means for better characterizing fractured geothermal reservoirs. This prediction motivated us to carry out flow tests at the Kamaishi Mine in Japan.

Self-potential (SP) observations associated with flow tests of boreholes have been conducted in various fields by now (e.g., [13-17]). Among them, SP observations designed for hydraulic characterization of groundwater aquifers were also conducted; the SP signals, which complement piezometric observations, were used to estimate the transmissivity of aquifers (e.g., [18-21]).

In the present study, we focus on the characterization of fractured reservoirs. First, SP transient signals expected for fractured reservoirs are explained based upon the results of numerical simulations $[8,10]$, and then SP data obtained from experiments at the Kamaishi Mine are described and interpretation of the data is discussed.

\section{SP Transients in Fractured Reservoirs}

2.1. Electrokinetic Coupling. The flow of a fluid through a porous medium will generate an electrical potential gradient (called the electrokinetic or streaming potential) along the flow path by the interaction of the moving pore fluid with the electrical double layer at the pore surface. This process is known as electrokinetic coupling. The general relations between the electric current density I and fluid volume flux J and the electric potential gradient $\nabla \phi$ and pore pressure gradient $(\nabla P-\rho \mathbf{g})$ forces are

$$
\begin{aligned}
& \mathbf{I}=-L_{\mathrm{ee}} \nabla \phi-L_{\mathrm{ev}}(\nabla P-\rho \mathbf{g}), \\
& \mathbf{J}=-L_{\mathrm{ve}} \nabla \phi-L_{\mathrm{vv}}(\nabla P-\rho \mathbf{g}),
\end{aligned}
$$

where the $L_{i j}$ are phenomenological coefficients (e.g., [22]). The first term on the right-hand side in (1) represents Ohm's law, and the second term in (2) represents Darcy's law.
The cross-coupling terms (with the $L_{\mathrm{ev}}$ and $L_{\mathrm{v}}$ coefficients) represent the electrokinetic effect, $L_{\mathrm{ev}}=L_{\mathrm{ve}}$ according to Onsagar's reciprocal relations. See the tutorial of this special issue for more details (Jouniaux and Ishido, this issue).

Based upon a capillary model, the above coefficients may be written as follows (e.g., [20]):

$$
\begin{gathered}
L_{\mathrm{ev}}=-\frac{\eta \varepsilon \zeta R_{\mathrm{ev}} G}{\tau \mu}, \\
L_{\mathrm{ee}}=\frac{\eta\left(\sigma+m^{-1} \Sigma_{s}\right)}{\tau},
\end{gathered}
$$

where $\eta=$ porosity, $\varepsilon=$ liquid-phase dielectric permittivity, $\zeta=$ zeta-potential, $R_{\mathrm{ev}}=$ "electrical relative permeability" for liquid/gas two-phase flow, $G=$ correction factor which becomes less than unity only if the hydraulic radius is comparable to the thickness of the electrical double layer, $\tau=$ square of tortuosity $\left(\tau=t^{2}\right), \mu=$ liquid-phase viscosity, $\sigma=$ electrical conductivity of pore fluid (two-phase mixture), $m=$ hydraulic radius of pores and/or cracks, which equals half of radius and aperture for pores with circular and slit-like crosssections, respectively, and $\Sigma_{s}=$ surface conductance.

Equation (1) describes the total current density, composed of a drag (convection) current density $\mathbf{I}_{\text {drag }}$ caused by charges moved by fluid flow and a conduction current density $\mathbf{I}_{\text {cond }}$ caused by electric conduction; hence,

$$
\mathbf{I}=\mathbf{I}_{\mathrm{cond}}+\mathbf{I}_{\mathrm{drag}},
$$

where

$$
\begin{gathered}
\mathbf{I}_{\text {cond }}=-L_{\mathrm{ee}} \nabla \phi, \\
\mathbf{I}_{\mathrm{drag}}=-L_{\mathrm{ev}}(\nabla P-\rho \mathbf{g}) .
\end{gathered}
$$

In the absence of external current sources, $\nabla \cdot \mathbf{I}=0$, so from (5),

$$
\nabla \cdot \mathbf{I}_{\mathrm{cond}}=-\nabla \cdot \mathbf{I}_{\mathrm{drag}}
$$

Equation (7) represents sources of conduction current that are required for the appearance of electrical potential.

In a homogeneous region with a homogeneous density $(\rho),(7)$ can be written as

$$
\nabla^{2} \phi=C \nabla^{2}(P-\rho g z)
$$

where $C$ is called the streaming potential coefficient, which is given as follows in case of liquid single-phase flow (and $G=1$ ),

$$
C=-\frac{L_{\mathrm{ev}}}{L_{\mathrm{ee}}}=\frac{\varepsilon \zeta}{\left(\sigma+m^{-1} \Sigma_{s}\right) \mu} .
$$

If the pore pressure change occurs within a finite homogeneous volume, the following relation between changes in $\phi$ (streaming potential) and $(P-\rho g z)$ (pressure) prevails:

$$
\Delta \phi=C \Delta(P-\rho g z)
$$


2.2. EKP Postprocessor. The cross-coupling term in (2) may be safely neglected for typical geologic situations, and Darcy's law alone may be used to model the hydraulic problem; it is not necessary to solve (1) and (2) simultaneously. A "postprocessor" may then be used to calculate the drag current $\left(\mathbf{I}_{\text {drag }}\right)$ from the results of an unsteady thermohydraulic reservoir simulation.

The "EKP-postprocessor" [9, 23] simulates electric potentials caused by subsurface fluid flow by a two-step process. First, it calculates the distribution of $L_{\mathrm{ev}}, L_{\mathrm{ee}}$, and $\mathbf{I}_{\text {drag }}$ from the reservoir-simulation results using the same spatial grid used for the reservoir simulation calculation (called the RSV grid hereafter). Next, the postprocessor calculates the electric potential $(\phi)$ distribution by solving the above Poisson equation (7) within a finite-difference grid that is usually much greater in spatial extent than the RSV grid (hereafter called the SP grid).

Within that portion of the SP grid overlapped by the RSV grid, the distribution of electrical conductivity is obtained directly from RSV grid values. Elsewhere within the SP grid, the electrical conductivity distribution is user specified and time invariant. Ordinarily, boundary conditions on the potential are zero normal gradients (Neumann condition) on the ground surface (upper surface) and zero potential (Dirichlet condition) along the bottom and vertical sides of the SP grid. Equation (7) is solved numerically using a Gauss-Seidel iteration procedure incorporating intermittent automatic optimization of the overrelaxation factor.

2.3. Model for Drag Current in MINC Media. The model which Ishido and Pritchett [8] adopted to calculate the drag current density in "MINC" media [4] amounts to the following:

$$
\mathbf{I}_{\text {drag }}=\mathbf{I}_{\mathbf{f}}+\mathbf{I}_{\mathbf{m}},
$$

with

$$
\begin{gathered}
\mathbf{I}_{\mathbf{f}}=\left[\frac{\varepsilon \zeta \eta R_{\mathrm{ev}} G(\nabla P-\rho \mathbf{g})}{\tau \mu}\right]_{f} \times \psi, \\
\mathbf{I}_{\mathbf{m}}=\left[\frac{\varepsilon \zeta \eta R_{\mathrm{ev}} G(\nabla P-\rho \mathbf{g})}{\tau \mu}\right]_{m} \times(1-\psi),
\end{gathered}
$$

where $\mathbf{I}_{\text {drag }}=$ total drag current density vector, $\mathbf{I}_{\mathbf{f}}=$ drag current density due to fracture zone effects, $\mathbf{I}_{\mathbf{m}}=$ drag current density due to matrix region effects, $\psi=$ fracture zone volume/total volume, and where subscript " $f$ " denotes conditions in the fracture zone, and subscript " $m$ " denotes "averaged" conditions in the matrix region (see [8] for further details). We detail now the SP transients related to a continuous injection, first for the MINC double-porosity media, and secondly for the reservoir model taking into account individual fractures and a borehole. We show that the characteristic behavior of SP predicted by the first model is present in the second model only if a skin zone with a lower streaming potential coefficient is assumed.

2.4. Pressure and SP Transients in MINC Media. Ishido and Pritchett [8] performed a pressure-transient simulation for a two-dimensional axisymmetric horizontal reservoir model. The formation is represented by an "MINC" double-porosity medium with the following properties: global permeability: $k=10^{-14} \mathrm{~m}^{2}$, fracture zone volume fraction: $\psi=0.1$, fracture zone porosity: $\eta_{f}=0.1$, matrix region porosity: $\eta_{m}=0.1$, matrix region permeability: $k_{m}=10^{-17} \mathrm{~m}^{2}$, and fracture spacing: $\lambda=10 \mathrm{~m}$. (In the present parameter setting, the fracture zone permeability is $k / \psi=10^{-13} \mathrm{~m}^{2}$, which is due to fractures occupying $10 \%$ volume of the fracture zone. The rest $90 \%$ volume is assumed to be of impermeable rock matrix.) The time required $\left(\tau_{\mathrm{pe}}=\eta_{m} \mu C_{t} \lambda^{2} / 10 k_{m}\right)$ for pressure equilibration between the fracture and matrix regions is $\sim 10^{4} \mathrm{sec}$ (here $C_{t}$ : total system (fluid plus rock) compressibility). The initial thermodynamic state is uniform (temperature $=200^{\circ} \mathrm{C}$ and pressure $=10 \mathrm{MPa}$ ). For the corresponding SP calculations shown in Figure 1, the reservoir fluid's $\mathrm{NaCl}$ concentration is assumed to be $0.02 \mathrm{~mol} / \mathrm{L}$, and the formation conductivity $L_{\mathrm{ee}}$ is assumed to be $0.03 \mathrm{~S} / \mathrm{m}$ (homogeneous).

Figure 1(a) shows semilog plots of changes in pressure and in SP due to continuous injection at 0.5 tons per hour per meter of reservoir thickness. The pressure transient at a point near the injection well shows behavior typical of a double-porosity medium; the late-time slope develops after the time required for pressure equilibrium within the matrix region $\tau_{\text {pe }}$ has elapsed.

The SP transient exhibits three segments. The drag current contribution through the matrix region is small at early times (up to $\sim 0.01$ day), so the slope is smaller than that at late times (after $\sim 0.1$ day), by the factor $\psi(=0.1)$. At intermediate times, SP changes rapidly with increasing involvement of matrix region. The time $\tau_{\mathrm{pe}}$ can be clearly identified at the intersection of the intermediate-time and late-time semilog straight lines (In Figure 1, the "observation" point is not located within the borehole, but $\sim 5$ meters away from the injection well. The reason for this is that in the case of "open hole," the SP change within the borehole does not show the typical behavior like that shown in Figure 1 since the pressure in the matrix region coincides with the borehole pressure even in early times as approaching the borehole. This topic will be explained in the next Section 2.5).

Figure 1(b) shows the ratio of SP changes to pressure changes for the results shown in Figure 1(a). In the case of the equivalent porous medium, relationship (10) is satisfied for the entire period, resulting in an almost constant ratio. In this plot, the difference between double-porosity and equivalent porous medium behavior is much more apparent and the time $\tau_{\text {pe }}$ is more evident than in a plot of SP change itself shown in Figure 1(a). The change-ratio plot has the additional advantage that, in real situations, pressure transient data suffer from fluctuations in the sandface flowrate, so it is often difficult to discern the three segments such as those shown in Figure 1(a). By contrast, the ratio of SP change to pressure change is insensitive to flowrate fluctuations, so a combination of pressure and SP measurements is expected to provide a more robust and reliable technique for fractured reservoir characterization. 


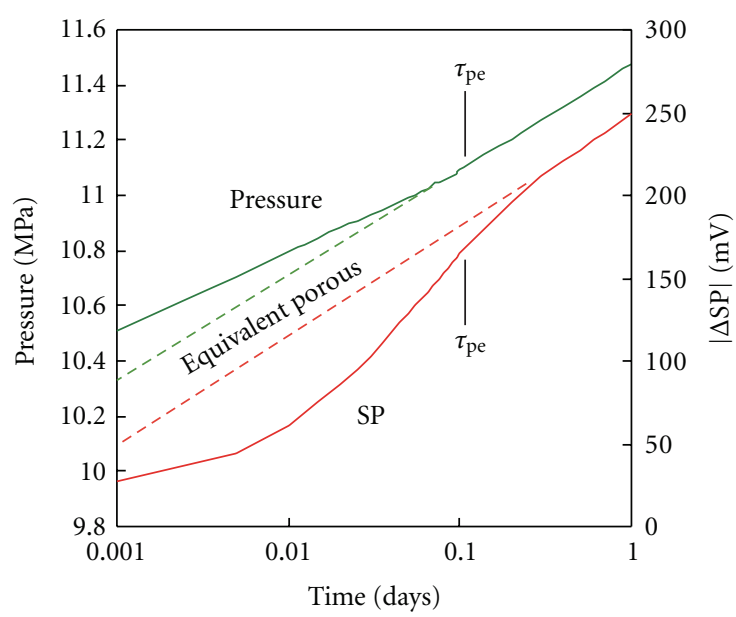

(a)

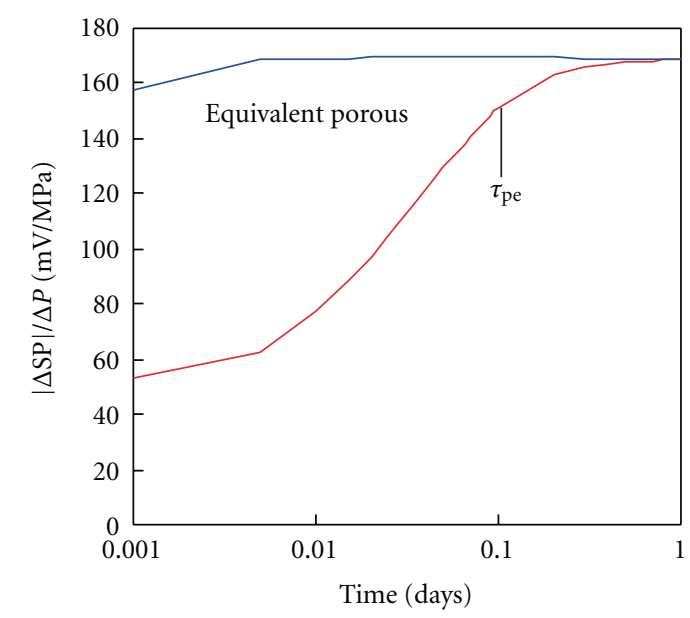

(b)

Figure 1: Results from the MINC double-porosity model. (a) Changes in pressure and SP at a point $\sim 5 \mathrm{~m}$ away from the injection well (open hole) during injection test for fractured medium (after [8]). The pressure and SP changes for the equivalent porous medium are shown by broken curves. (b) Plot of the ratio of SP change to pressure change converted from the pressure and SP changes shown in (a).

2.5. Near-Field Effects. In the calculations described in the previous section, the "near-field" effects around a borehole were not considered. Ishido et al. [10] constructed a reservoir model to treat a borehole and individual fractures explicitly instead of using the MINC double-porosity representation (Figure 2). The model is axisymmetric, eight meters thick, and of $1 \mathrm{~km}$ horizontal extent (radius). Five equally spaced horizontal fractures intersect the borehole located along the axis of symmetry (only two fractures are drawn schematically in Figure 2). Sufficiently fine block spacing was adopted near the borehole (radius of 0.075 meter) to represent the well casing. Fine block spacing was also used for the host rock (matrix) region close to the fracture zone so as to resolve the high electrical potential gradients there. Here, only the

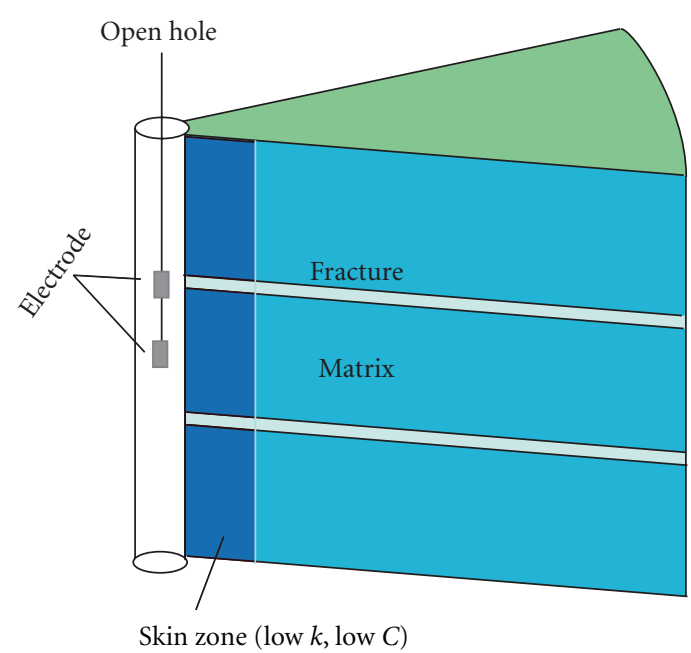

Figure 2: "Open hole" model used for numerical simulation of SP transient near a borehole (after [10]). In "open (skin)" case, a skin zone of low permeability and low magnitude of streaming potential coefficient is assumed for the matrix region.

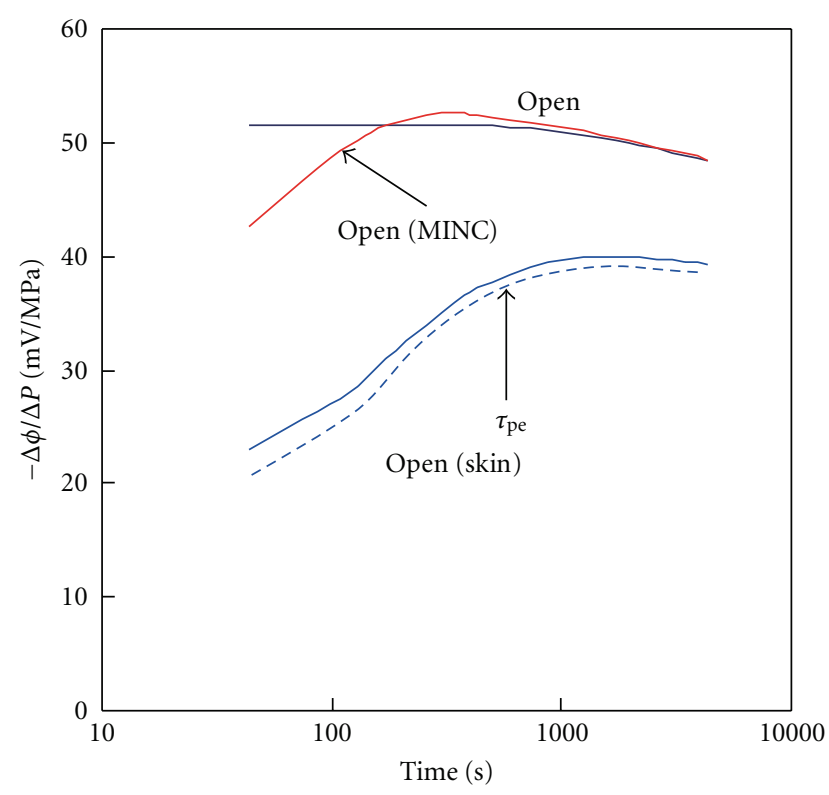

FIGURE 3: The ratio of SP change to pressure change as a function of time (after [10]). Results from the reservoir model, for (a) open hole homogeneous case "open" and (b) open hole with a skin-zone case "open (skin)"; (c) Result from the equivalent MINC doubleporosity model "open (MINC)."

results for open hole cases are explained. See [10] for the results of cased wells.

The formation properties are: fracture zone permeability: $k_{f}=10^{-12} \mathrm{~m}^{2}$, fracture zone thickness $=0.01 \mathrm{~m}$, fracture zone porosity: $\eta_{f}=0.5$, host rock porosity: $\eta_{m}=0.01$, host rock permeability: $k_{m}=10^{-18} \mathrm{~m}^{2}$, and fracture spacing: $\lambda=1 \mathrm{~m}$. The time required $\left(\tau_{\mathrm{pe}}\right)$ for pressure equilibration between the fracture and host rock regions is $\sim 500 \mathrm{sec}$. The initial thermodynamic state is uniform (temperature $=45^{\circ} \mathrm{C}$ 


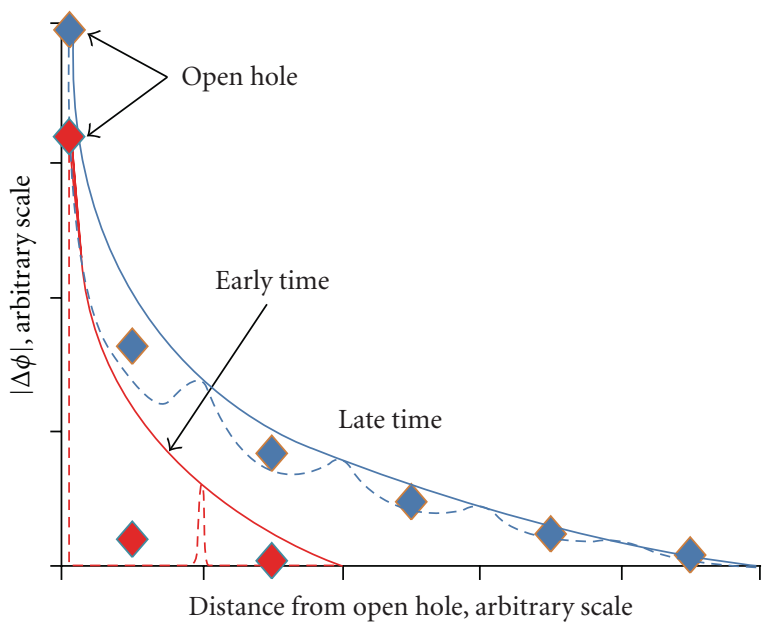

FIGURE 4: Results from the reservoir model taking into account individual fractures and a borehole. Schematic diagram showing the distribution of "microscopic" potential in the fracture (solid lines) and matrix (broken lines) regions in the reservoir, which is equal to change in local pressure multiplied by the streaming potential coefficient $C$ (here homogeneous $C$ is assumed for simplicity). "Macroscopic" potential $(\checkmark)$ calculated for MINC-medium blocks corresponds to an averaged potential over the fracture and matrix regions (after [10]).

and pressure $=10 \mathrm{MPa})$. Fluid production takes place from the borehole with a constant pressure drawdown of $1 \mathrm{MPa}$. For the corresponding SP calculations, the reservoir fluid's $\mathrm{NaCl}$ concentration is assumed to be $0.005 \mathrm{~mol} / \mathrm{L}$, and the streaming potential coefficient is uniform throughout the fracture and host rock regions.

In the "open hole" cases, the SP change that would be measured by electrode(s) installed within the borehole (Figure 2) is calculated, and then the ratio of SP change to pressure change is plotted as a function of time (Figure 3 ). As Figure 3 shows, the "open" ratio is almost constant with time, which is similar to the "equivalent porous medium" behavior shown in Figure 1(b) and does not exhibit any characteristic fractured reservoir behavior.

But if a skin zone in which the streaming potential coefficient is much smaller than that of the outer matrix region is present, the typical double-porosity behavior appears in the plot ("open (skin)" curves in Figure 3). Although the SP change magnitude is independent of the location of the electrode for the case without a skin zone, the SP change is slightly smaller at the electrode located at the matrix region for the case with skin zone as shown by the broken line in Figure 3.

In Figure 3, also shown (in red) is the result for a case in which the reservoir is represented by "equivalent" MINC double-porosity medium. In this "open (MINC)" case, the $\mathrm{SP} /$ pressure change ratio exhibits similar behavior as that of "open" case. This is because the present scheme used by the EKP postprocessor to calculate SP in an MINC medium gives an "averaged" potential over the fracture and matrix regions.

Figure 4 illustrates the spatial distribution of "microscopic" potential, which is the streaming potential coefficient multiplied by local pressure change, in the fracture and matrix regions around an open hole. Along the wall of the open hole, the local pressures in both the fracture and matrix regions are equal to the borehole pressure, so the microscopic potentials in both the regions converge as the borehole is approached. This is the reason why the SP change is in proportion to the pressure change within the borehole in the "open" case. However, in the "open (skin)" case, substantial drag current is not induced in the matrix region, while a large pressure gradient remains within the skin zone. So, SP change within the borehole is brought about solely by the drag current induced in the fracture zone, resulting in smaller SP change magnitude at early times.

\section{Field Experiments at the Kamaishi Mine}

The Kamaishi Mine had been one of the largest mines in Japan, which produced copper and iron ore over 130 years since 1857 (see the inset of Figure 5 for its location). The total amount of ore mined during this period is about 70 million tons. The galleries of $\sim 140 \mathrm{~km}$ in total length were made mainly in hard rocks composed of Kitakami Paleozoic granitic rocks, Mesozoic granitic intrusions, and skarn ore deposits formed at the contacts of the intrusions. During the last more than two decades, the mine has been diversifying from mining into underground research sites for developing new methods of geological and geophysical studies and for rock mechanics, hydrology, and so forth (e.g., [24]). In this section, we describe the experiments performed by inducing a fluid flow from horizontal wells, which causes pressure disturbance along the entire borehole both in the fracture and host rock matrix regions.

3.1. Outline of Measurements. We carried out flow tests and pressure and SP measurements in two open holes (KF-1 and KF-3) which were drilled nearly horizontally from the wall of one of the levels into the surrounding granodiorite body (both wells were drilled in the direction $\sim 15^{\circ}$ from the direction of the tunnel on the horizontal plane as shown in Figure 5). Both wells maintain stable pressures of about five bars under shut-in conditions, so that flow tests may be carried out by simply opening and closing the wellhead valves. After preliminary experiments in 2005 and 2006 [25], we installed twelve custom-made silver-silver chloride electrodes in each of the two wells in 2007 [26].

To reduce flow effects on measuring electrodes, each silver-silver chloride electrode was installed in a container made of hard plastic tube, the upstream and downstream ends of which were closed and open (via sponge), respectively (Figure 6). The voltages between each electrode and the reference (the location of which is shown in Figure 5) and the pressure and flow rate of the two wells were recorded with two data loggers Campbell Scientific's CR5000. The valve operation of wells KF-1 and KF-3 was automatically controlled, and all measuring equipments were powersupplied by a few car batteries, which were fully charged at maintenance time. One unit of the experiment was carried out in two days; KF-1 was opened for one hour on the first 


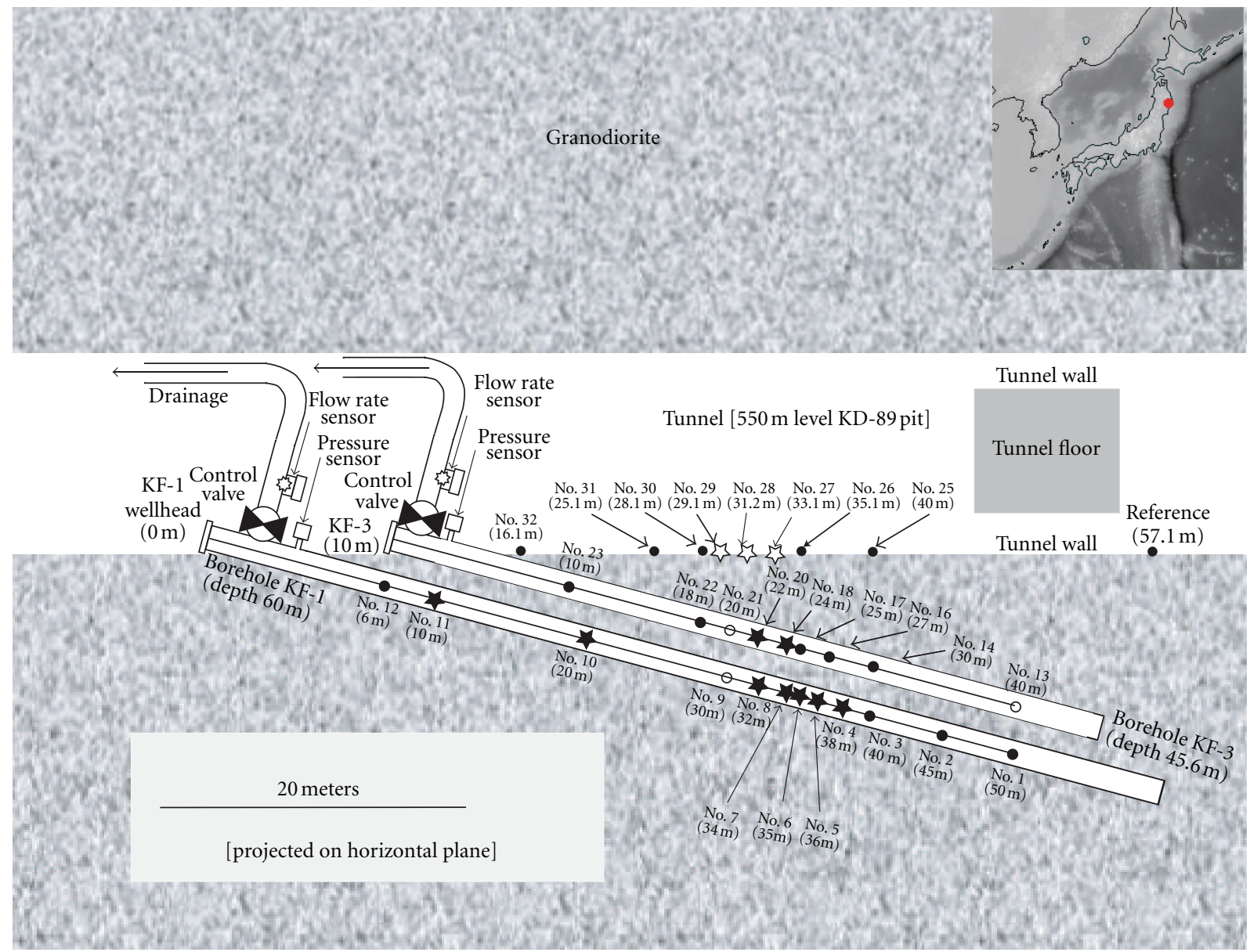

Figure 5: Plan view of experimental setup of pressure and SP measurements at the Kamaishi Mine in April-June 2007. Ag-AgCl electrodes No. 1 to 12 and No. 13 to 24 were installed in wells KF-1 and KF-3, respectively. Ag-AgCl electrodes were also installed on the tunnel floor at eight points No. 25 to 32 and at the reference point. Locations of the borehole electrodes near permeable fractures which showed distinguishable behaviors from those of other electrodes are shown by solid asterisks. Locations of tunnel floor electrodes which showed SP changes corresponding to the flow from KF-1 or KF-3 are shown by open asterisks. Locations of other electrodes are shown by solid or open circles (open circles show the locations of electrodes which were sometimes unstable during the period from April to June 2007).

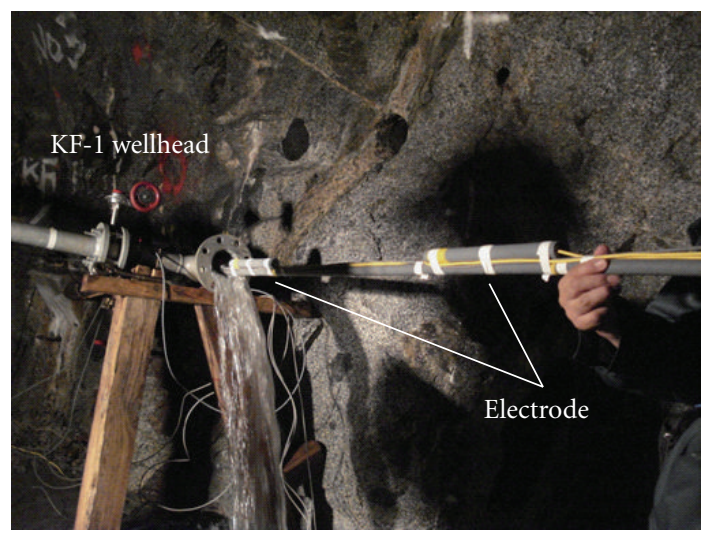

FIGURE 6: Photo of installation of custom-made $\mathrm{Ag}-\mathrm{AgCl}$ electrode which is contained in a plastic tube into well KF-1. day, and then KF-3 was opened for one hour on the second day. We repeated this procedure as many as possible with various valve openings in April through June 2007.

Examples of the test results are shown in Figure 7. In Figure 7(a), the results are shown for an experiment when well KF-1 was flowing on May 13. The two wells are connected to each other through permeable fractures, so KF3 pressure also substantially decreased. Corresponding to the pressure decreases, SP in both wells KF-1 and KF-3 increased several millivolts. Concerning the SP changes on the tunnel (level) floor, their appearance is restricted to an interval of $\sim 5$ meters near the fracture zones; only three electrodes nos. 27, 28, and 29 (see Figure 5 for the locations) showed a few millivolts decrease and increase corresponding to the start and stop of the flow, respectively (in Figure 7, the data of nos. 27 and 29 showing substantial changes and the data of no. 25 

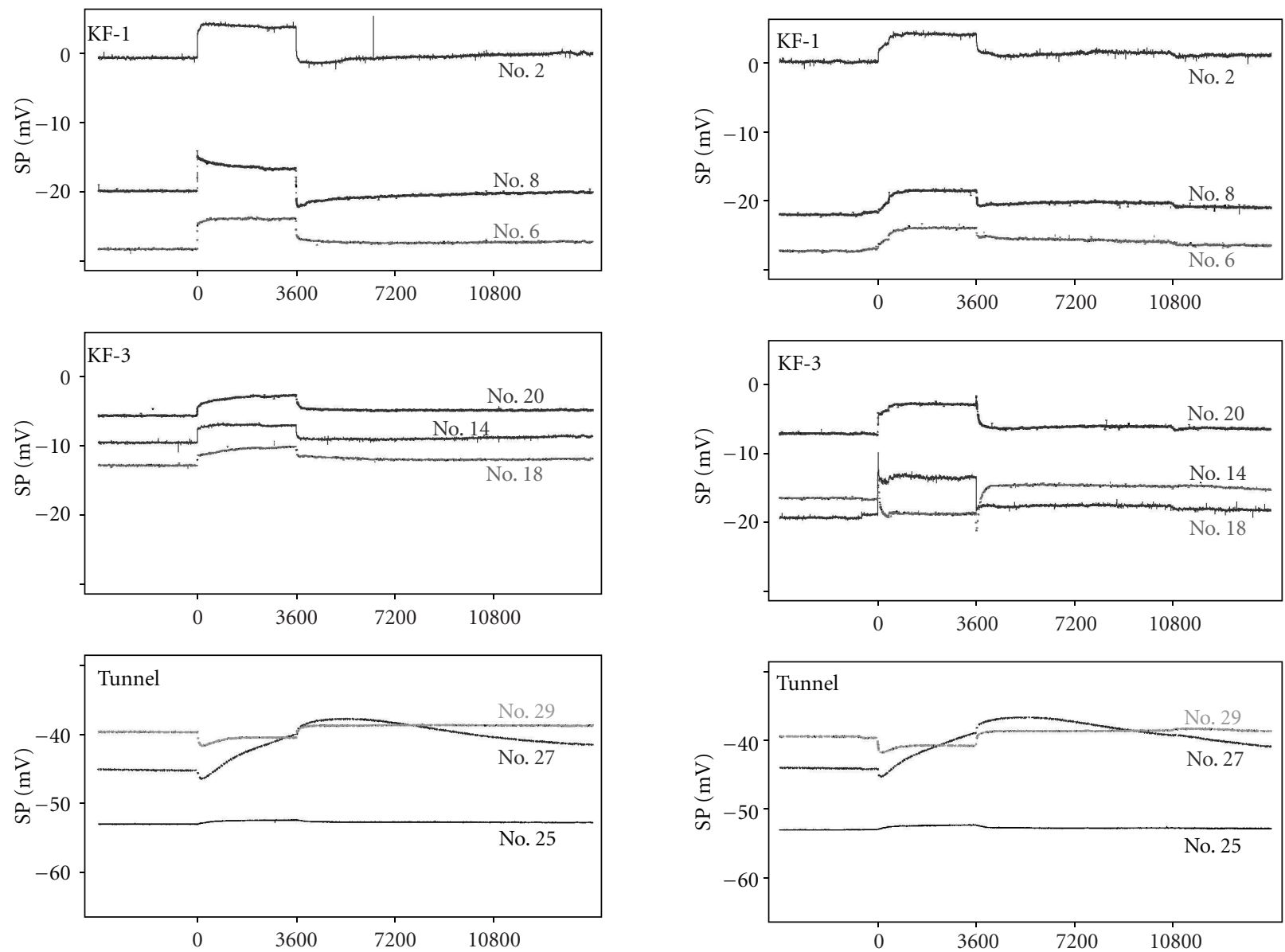

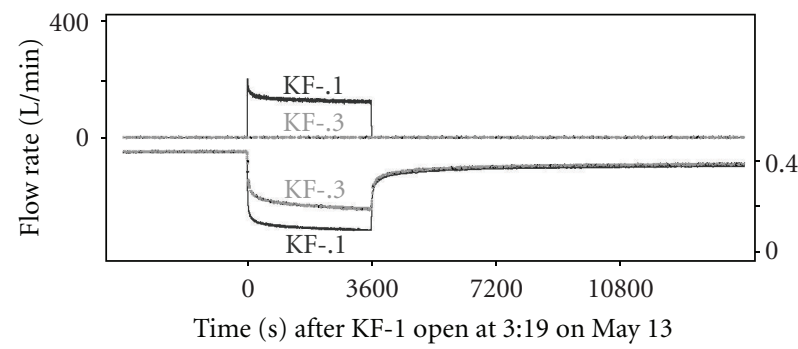

(a)

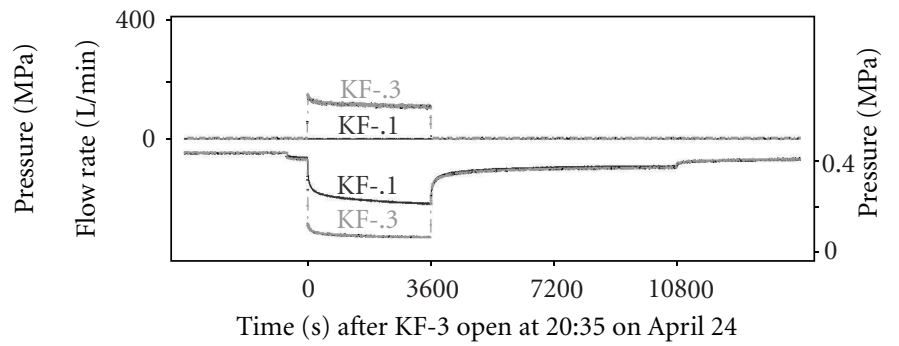

(b)

FIGURE 7: Recorded data during one cycle of valve operation. Changes in SP in wells KF-1 and KF-3 and on the tunnel floor are shown for the valve opening of KF-1 (a) and KF-3 (b). Also shown are the recorded pressure and flow rate of wells KF-1 and KF-3 (bottom).

showing only a tiny fluctuation are shown). In Figure 7(b), the results are shown for an experiment when well KF-3 was flowing on April 24. Similar SP changes to those when KF-1 was flowing were observed.

As seen in Figure 7, some of the electrodes installed in the flowing well showed spike-like changes at the start and stop of the flow, which seem to correlate with sudden flow rate changes and influence the successive SP changes. So hereafter, we focus on the SP data measured in "observation" wells, that is, KF-3 and KF- 1 corresponding to the flow of KF-1 and KF3 respectively, which are thought to be less influenced by the flow effects on the electrodes. In the next section, three KF-3 observations associated with KF-1 valve opening (70\%) for one hour on April 23, May 3, and May 13 and three KF-1 observations associated with KF-3 valve opening (70\%) for one hour on April 24, May 4, and May 14 will be shown. Among these, the data from experiments on April 24 and May 13 are the same as those shown in Figure 7.

3.2. Results. Figure 8 shows three records (with one-second sampling interval) from each of four electrodes in KF-3 (observation well) associated with KF-1 valve opening on April 23, May 3, and May 13. As seen in the figure, the 

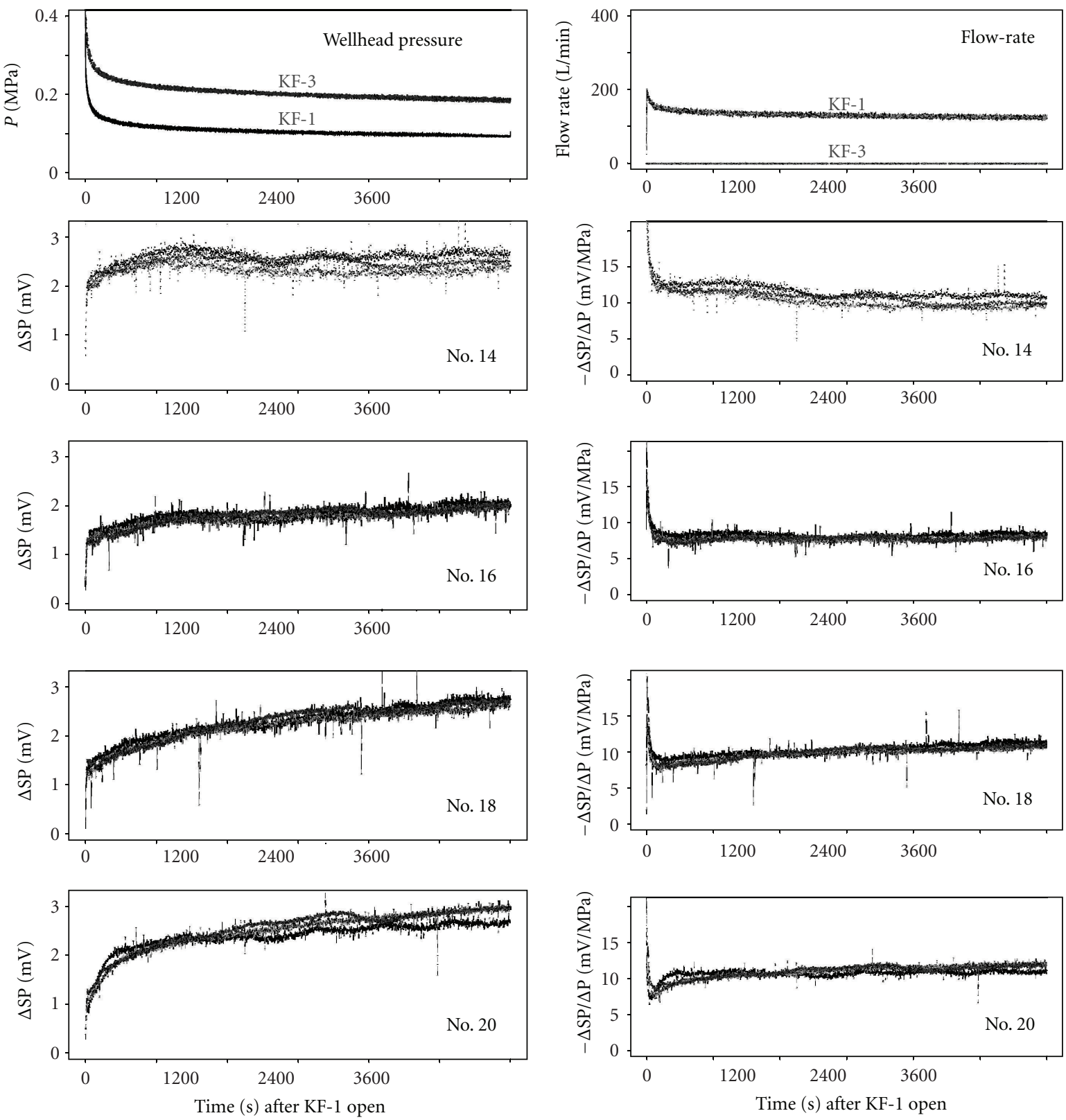

FIGURE 8: Changes in SP in KF-3 (observation well) for three repeated KF-1 valve operations. Ratio of SP change to pressure change is also shown on the right. Both of $\Delta \mathrm{SP}$ and $\Delta \mathrm{SP} / \Delta P$ are fairly reproducible.

differences among the three records are not so small compared to those of pressure and flow rate histories, but overall trends are fairly reproducible both for SP change $(\Delta \mathrm{SP})$ and the ratio of SP change to pressure change $(\Delta S \mathrm{P} / \Delta P)$. The $\Delta \mathrm{SP} / \Delta P$ corresponds to the streaming potential coefficient, which is estimated to be around $-10 \mathrm{mV} / \mathrm{MPa}$ from the records at later times. During the first $\sim 100$ seconds, the magnitude of $\Delta \mathrm{SP} / \Delta P$ rapidly decreased from the initial values of around $20 \mathrm{mV} / \mathrm{MPa}$ to $\sim 10 \mathrm{mV} / \mathrm{MPa}$. This rapid change is thought to be caused by (1) electrode drifts induced by the flow start, which were small in the observation well but not negligible compared to the small signals at early times and (2) uncertainty involved in the evaluation of SP values just before the valve opening. We usually determined "static" SP values from the average values over intervals between 25 and 15 minutes before the valve opening. In Figure 9, comparison between the results using this average "static" value and an instantaneous "static" value just at the valve opening is shown. The differences between the two curves are relatively minor even for earlier times than 100 seconds. However, in cases such as the experiment on April 24 shown in Figure 7, a small pressure down occurred before 10 minutes before the flow start, which was caused by a faint unexpected opening of the automatically controlled wellhead valve when power was supplied for operational preparation. In such cases, SP was disturbed from 10 minutes before the 


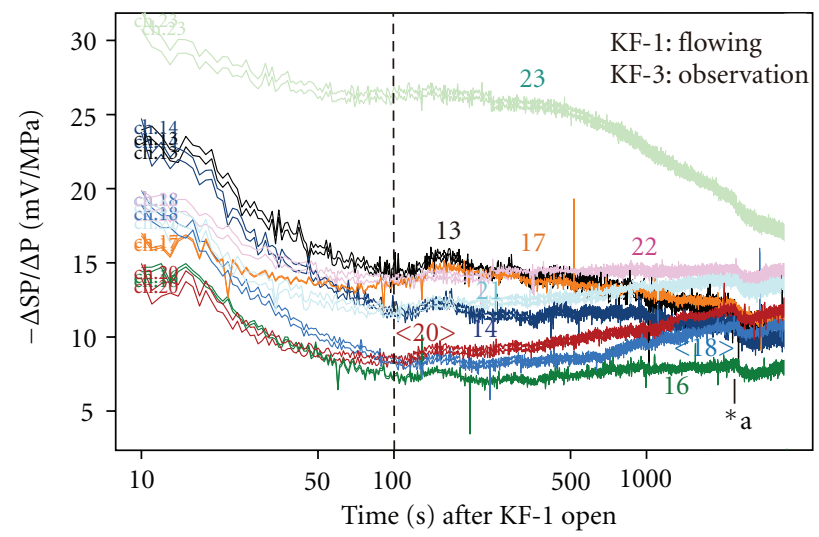

(a)

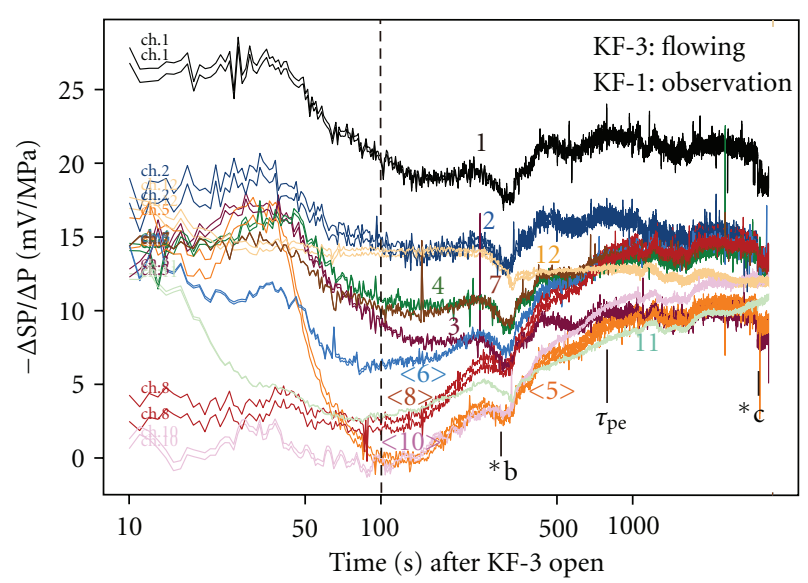

(b)

Figure 9: Ratio of SP change to pressure change as a function of time for KF-3 observation on May 13, 2007 (a) and KF-1 observation on May 14, 2007 (b). Two records for each electrode are drawn with different "static" SP values: one is with an average value over interval between 25 and 15 minutes before the valve opening and another is with an instantaneous value just at the valve opening at time zero. The differences between the two curves are relatively small even for earlier times than 100 seconds in the results shown here. At times pointed by ${ }^{*} \mathrm{a}$, $*$ b, and ${ }^{*}$ c, all electrodes in wells KF-1 and KF-3 were suffered from a shift or a short-term disturbance. If the "double-porosity" model is applied to the KF-1 observation, the time required for pressure equilibration between the fracture and matrix regions is estimated as $\tau_{\mathrm{pe}} \sim 800$ seconds.

flow start at $t=0$, and the difference between the two results with "static" values determined from the instantaneous (at $t=0$ ) and average (between $t=-25$ and -15 minutes) values became significant for early times. While the SP changes remained below $\sim 1 \mathrm{mV}$, these uncertainties would easily bring about factor 2 to 3 uncertainty in $\Delta S P / \Delta P$ values. So we will disregard the first 100 seconds of $-\Delta S P / \Delta P$ plots in the following discussion.

In Figures 9 and $10, \Delta \mathrm{SP} / \Delta P$ is plotted as a function of logarithm of time. Figure 10 shows the records of selected electrodes for three KF-3 observations on the left and three KF-1 observations on the right. As seen in the figure, the results for KF-3 observations are fairly reproducible. Three different behaviors are recognized: the first one shows a stable or small decreasing trend observed at electrodes located at host rock zone without permeable fractures (no. 14 and no. 16), the second one shows an increasing trend observed at electrodes located near permeable fractures (no. 18 and no. 20 ), and the third one shows a high magnitude at early times and decreasing trend after $\sim 400$ seconds observed at the shallowest electrode no. 23.

In contrast to the KF-3 observations, the reproducibility is not good for the KF-1 observations. This is mainly due to a shift (equivalent to $\sim 1 \mathrm{mV}$ positive SP change) appearing around 300-400 seconds for all electrodes except the shallowest electrode no. 12 in the first and second observations. A "V"-shaped change appeared also around 300-400 seconds for all electrodes in the third observation. Such shift or rapid change did not appear for the electrodes installed on the tunnel floor (eight electrodes nos. 25-32), and the occurrence time was not correlated with time of a day (the first, second, and third ones were at 20:40 on April 24, at 12:08 on May 4, and at 3:35 on May 14, resp.), and a possibility of external noise source due to working activity of the mine is low. In addition, no anomalous change is present in pressure and flow rate histories in the three observations (see Figure 7 for April 24 data). We have not understood the cause of these anomalous changes yet. However, comparing the earlier records than 300-400 seconds of the three observations, the changing patterns are quite similar to each other. This is also true for the later records than 300-400 seconds.

Records of all electrodes for the third KF-1 observation are shown in Figure 9. The behaviors can be divided into three groups; the difference between the earlier and later portions than 300-400 seconds is relatively small for the first group to which electrodes located at host rock zone without permeable fractures belong (nos. 1, 2, 3, and 12). The magnitude is small at early times, increasing up to $\sim 800$ seconds, and stable afterwards for the second group to which electrodes located near permeable fractures belong (nos. 5, 6,8 , and 10). The third group (electrodes nos. 4, 7, and 11) shows an intermediate behavior between those of the first and second groups. The observed $\Delta \mathrm{SP} / \Delta P$ is almost constant with time in the host rock zone but exhibits temporal variation near the fractures, which looks like the "doubleporosity behavior" predicted by Ishido and Pritchett [8]. If their prediction is applied, the time required for pressure equilibration $\left(\tau_{\mathrm{pe}}\right)$ between the fracture and matrix regions will be identified at the intersection of the intermediate time increasing and late-time stable trends seen in the changeratio plots; $\tau_{\text {pe }}$ is estimated to be about 800 seconds for KF-1 observations.

3.3. Interpretation. Figure 11 shows the resistivity distribution around wells KF-1 and KF-3 [11]. The main permeable fracture zone located around 30 meters depth of KF-1 

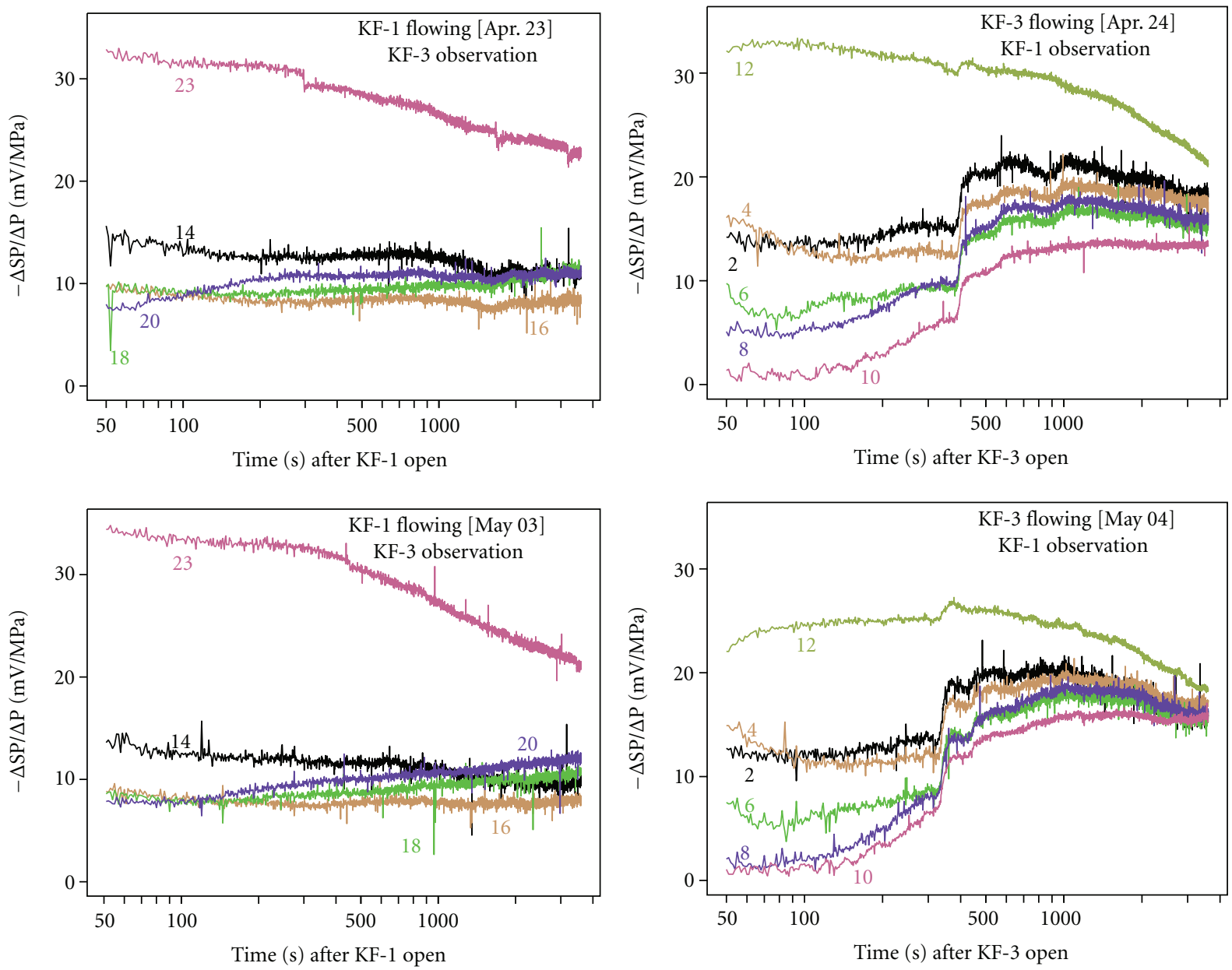

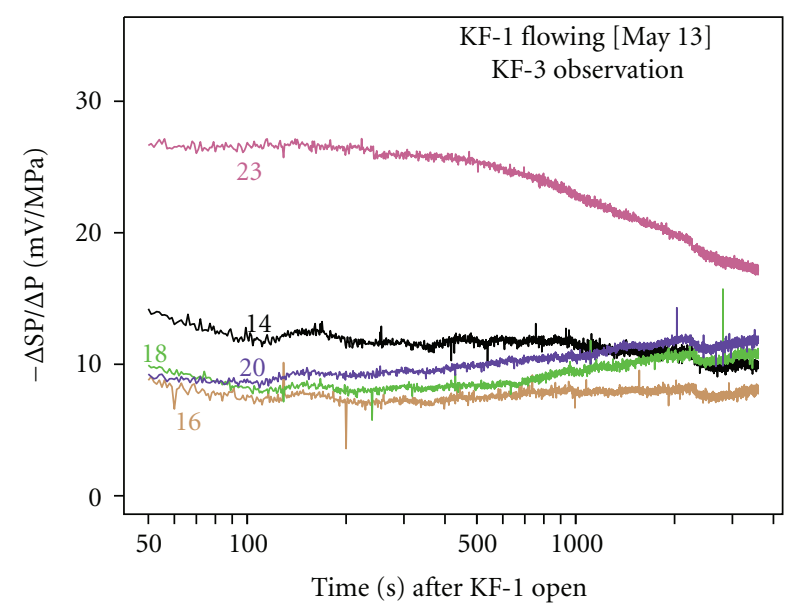

(a)

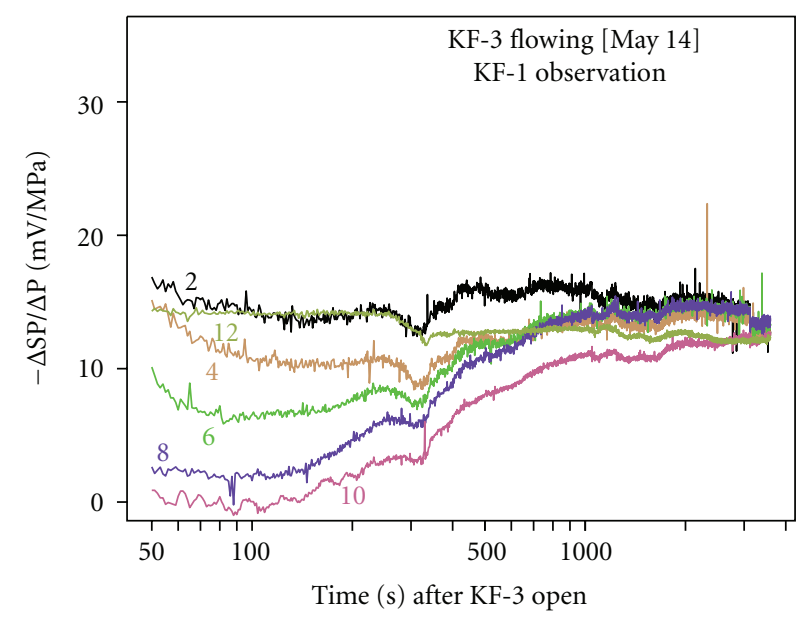

(b)

FIGURE 10: Ratio of SP change to pressure change as a function of time for KF-3 observations associated with three KF-1 valve opening (a) and for KF-1 observations associated with three KF-3 valve opening (b). The start of pressure drop is at time zero.

[12] is clearly delineated as a relatively low-resistivity zone $(\sim 800 \Omega \mathrm{m})$ within high-resistive $(\sim 4000 \Omega \mathrm{m})$ rocks. The extent of this low-resistive zone is well correlated with lowvelocity zone revealed by seismic tomography measurements [27]. The locations of KF-1 electrodes of the first, second, and third groups mentioned above are indicated by circle, yellow-filled asterisk, and white-filled asterisk, respectively, in Figure 11. The locations of KF-3 electrodes showing increasing trend and other KF-3 electrodes are indicated by white-filled asterisk and circle, respectively, in Figure 11. 


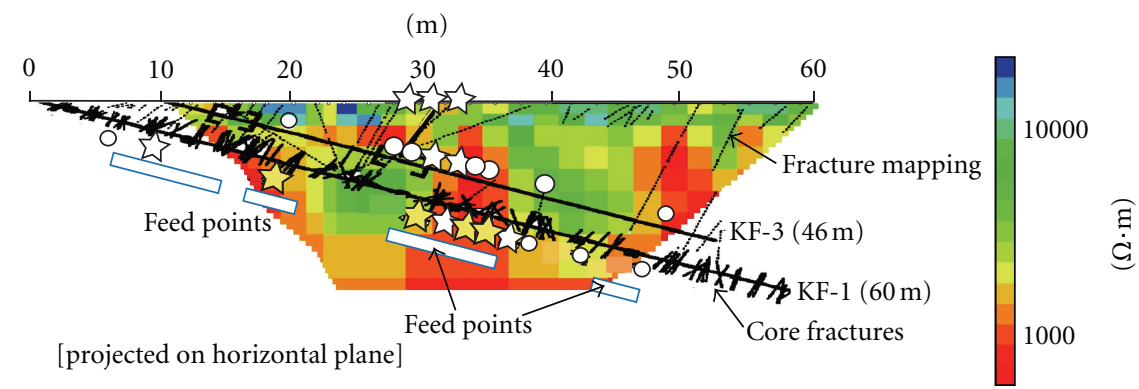

FiguRe 11: Electrical resistivity distribution around wells KF-1 and KF-3 (after [11]). Also shown are traces of major fractures, directions of core fractures, and locations of fluid feed points for KF-1 (e.g., [12]). Locations of KF-1 electrodes (nos. 5, 6, 8, and 10) showing typical fractured rock behavior in Figures 9 and 10 are denoted by yellow-filled asterisks, and those of KF-3 electrodes (nos. 18, 20) showing increasing trend are shown by white-filled asterisks. Locations of electrodes showing almost constant $\Delta \mathrm{SP} / \Delta P$ with time are shown by circles.

Different $\triangle \mathrm{SP} / \triangle P$ behaviors observed at lowpermeability host rock and permeable fractured rock regions can be explained by a conceptual model shown in Figure 12. After valve opening of KF-1 (KF-3), pressure drawdown immediately propagates to nearby observation well KF-3 (KF-1) through permeable fractures. Due to substantial pressure drawdown, radial fluid flow toward the wellbore also takes place in host rocks surrounding the observation well (point "A" in Figure 12), which makes $\Delta \mathrm{SP} / \Delta P$ equal to the streaming potential coefficient $(C)$ from early times. In fractured rocks, pressure within the matrix region remains near the initial pressure at early times. So large pressure gradient is created in the matrix region near the fractures and/or the wellbore (point B in Figure 12). As shown in Section 2.5, the magnitude of $\Delta \mathrm{SP} / \Delta P$ is modeled to be quite large from early times and constant with time thereafter if the matrix region's $C$ is assumed to be homogeneous. But if $C$ in the near surface skin zone is assumed significantly smaller in magnitude than that in the interior of the matrix region, $|\Delta \mathrm{SP} / \Delta P|$ is modeled to remain at quite small value at early times.

As shown in Figure 11, quite large contrast of resistivity is present between the main fracture and the surrounding host rock regions. This might bring about difference in the streaming potential coefficient between the fracture and host rock regions. We need to take into account the presence of such heterogeneity in addition to that from EDZ (excavationdamaged zone), which is thought to have $\sim 1 \mathrm{~m}$ thickness around the tunnel wall (e.g., [24]), in future studies.

If we apply the "double-porosity" model mentioned in Section 2 to the KF-1 observations shown in Figure 9, the fracture spacing is estimated to be a few meters based on the $\tau_{\text {pe }}$ value of $\sim 800$ seconds and the matrix rock permeability of several microdarcies $\left(10^{-18} \mathrm{~m}^{2}\right)$ deduced by testing core samples. This is in the range of spacing of permeable fractures estimated for the fracture zone of KF1 from detailed geological and hydrological observations (e.g., $[12,28]$ ). The ratio of SP change to pressure change corresponds to the streaming potential coefficient $C$ and is $-7 \sim-20 \mathrm{mV} / \mathrm{MPa}$ as seen in Figure 9 for the nonfractured host rock region. This value is in the range of $C$ measured for an intact granite sample in dilute solutions [29]. As for the fracture zone, the final asymptotic value is also about $-10 \mathrm{mV} / \mathrm{MPa}$. This suggests that the contribution of drag current through the matrix region dominates under steadystate conditions.

We measured the zeta potential $(-20 \mathrm{mV})$ for a crushed sample of granodiorite rock cored near the wellheads of KF-1 and KF-3 under room temperatures using the water discharged from the wells $\left(T \sim 11^{\circ} \mathrm{C}, \mathrm{pH} \sim 9\right.$ and electrical conductivity $\sim 0.85 \times 10^{-4} \mathrm{~S} / \mathrm{m}$ at sampling site; major chemical components are $\mathrm{Na}^{+} \sim 6, \mathrm{Ca}^{++} \sim 9, \mathrm{Cl}^{-} \sim$ $2.5, \mathrm{HCO}_{3}{ }^{-} \sim 33$, and $\left.\mathrm{SO}_{4} \sim 7 \mathrm{mg} / \mathrm{L}\right)$. If we assume the zeta potential of in situ intact host rock is also around $-20 \mathrm{mV}$, we can estimate the in situ streaming potential coefficient by using (9). Unfortunately, we do not know the surface conductivity, but its contribution must be significant since the in situ bulk conductivity $(1 / 800 \sim 1 / 4000 \mathrm{~S} / \mathrm{m})$ is even higher than the pore fluid conductivity itself. If we use the observed bulk conductivity in (9), the streaming potential coefficient is given as $C=(\eta / \tau)(\varepsilon \zeta / \mu) / L_{\mathrm{ee}}$. By substituting $\zeta=-0.02 \mathrm{~V}, \varepsilon=83.6 \times 8.85 \times 10^{-12} \mathrm{~F} / \mathrm{m}, \mu=0.00127 \mathrm{~Pa}-\mathrm{s}$, and $L_{\mathrm{ee}}=2.5 \times 10^{-4} \mathrm{~S} / \mathrm{m}$ into this equation, $C=-(\eta / \tau) \times$ $4.7 \times 10^{4} \mathrm{mV} / \mathrm{MPa}$. If we assume term $\eta / \tau\left(=F^{-1}\right.$, here $F$ is the electrical formation factor) to be 0.0002 , which is thought to be in the range of $F^{-1}$ for intact crystalline rocks (e.g., [29]), $C$ becomes about $-10 \mathrm{mV} / \mathrm{MPa}$, which is comparable to the observed "in situ" values: $-7 \sim-20 \mathrm{mV} / \mathrm{MPa}$. Using this value for $F^{-1}$, we can also estimate the surface conductivity $\left(m^{-1} \Sigma_{s}\right)$ in $(4)$ or $(9)$ to be around $1.25 \mathrm{~S} / \mathrm{m}$.

\section{Concluding Remarks}

We have carried out continuous pressure and SP monitoring in KF-1 and KF-3 wells at the Kamaishi Mine by inducing a fluid flow from these wells, which caused pressure disturbance in the surrounding formation along the entire borehole. The observed ratio of SP change to pressure change associated with the fluid flow showed different behaviors between intact host rock and fractured rock regions. In open holes, the appearance of double-porosity behavior is modeled to depend on whether or not a skin zone or some heterogeneity in the streaming potential coefficient is present. It may be that skin zones will be found in most open 


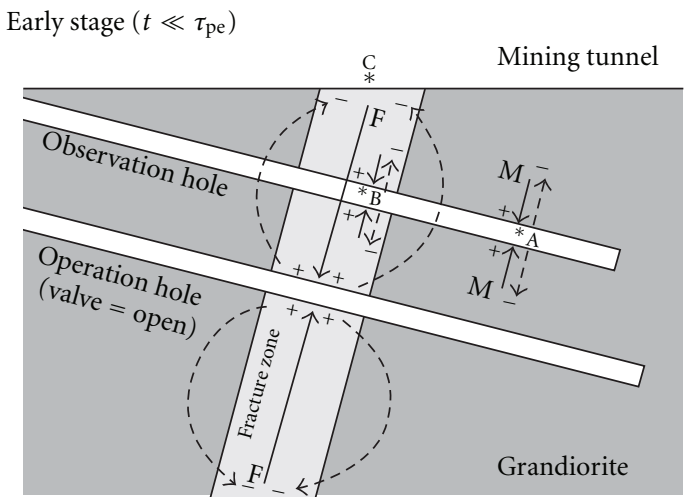

Later stage $\left(t>\tau_{\mathrm{pe}}\right)$

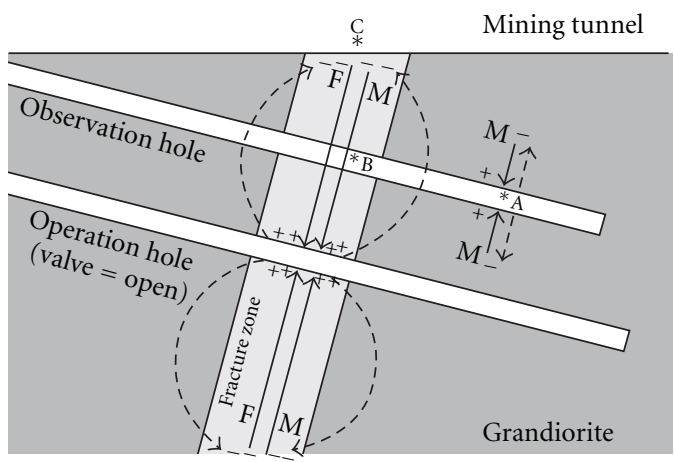

Figure 12: A conceptual model for different behaviors of SP changes at the country rock (A) and fractured rock (B) regions, and on the tunnel wall/floor (C). The drag current (solid line) flows only through the fracture $(\mathrm{F})$ region at early times and through both of the fracture $(\mathrm{F})$ and matrix $(\mathrm{M})$ regions at later times than the time required for pressure equilibrium between the fracture and matrix regions. The conduction current (broken line) flows to compensate the resulting charge separation. The model is drawn for a case that KF-3 and KF-1 are observation and flowing wells, respectively; but the model is also applicable to a case that KF-1 and KF-3 are observation and flowing wells, respectively.

hole completions, so measurements of this type in open holes are likely to be useful to deduce the time required for pressure equilibrium between the fractures and the rock matrix.

When the observation is carried out using cased wells, the hydraulic communication between the borehole and the formation should be restricted to the fracture region. Direct hydraulic contact between the borehole and matrix region is prevented by solid casing and cementing [10]. To detect microscopic $\Delta \mathrm{SP} / \Delta P$, an electrode array installed outside the insulated casing is desirable. Such observations were reported from an oil field [17]. Downhole SP measurement is thought to be a promising monitoring technique in various applications (e.g., $[30,31])$. How to equip observation and/or production/injection wells with appropriate electrodes is very important for these applications. To detect macroscopic $\Delta \mathrm{SP} / \Delta P$ such as predicted by Ishido and Pritchett $[8]$, the conductive casing itself (with electrical continuity extending over a distance longer than the typical fracture spacing) can be used as an electrode. Surface SP measurements (e.g., $[14,21]$ ) detected SP changes which were generated at the reservoir depths and transferred to the earth's surface through the conductive well casing. Thus surface SP measurement around a wellhead is also promising to detect macroscopic $\Delta \mathrm{SP} / \Delta P$ if the hydraulic communication between the borehole and the formation is restricted to the fracture region.

\section{Acknowledgments}

The present study was supported by basic research fund from The Institute for Geo-Resources and Environment, AIST. The authors greatly acknowledge the generous courtesy received from Kamaishi Mine Co., Ltd. and Nittetsu Mining Consultants Co., Ltd. during the field experiments. They express special thanks to the Kamaishi city where they stayed during the field surveys. They also wish the city recovers as soon as possible from the tsunami damages caused by the 2011 off the Pacific Coast of Tohoku Earthquake. The associate editor L. Jouniaux and two anonymous reviewers are acknowledged for helpful comments to improve the paper.

\section{References}

[1] J. W. Pritchett and S. K. Garg, "On similitude, heat conduction, and two-phase flow in fractured porous media," in Proceedings of the 15th Stanford Workshop on Geothermal Reservoir Engineering, 1990.

[2] J. E. Warren and P. J. Root, "The behavior of naturally fractured reservoirs," Society of Petroleum Engineers Journal, vol. 3, pp. 245-255, 1963.

[3] H. Kazemi, "Pressure transient analysis of naturally fractured reservoirs with uniform fracture distribution," Society of Petroleum Engineers Journal, vol. 9, pp. 451-462, 1969.

[4] K. Pruess and T. N. Narasimhan, "A practical method for modeling fluid and heat flow in fractured porous media," in Proceedings of the the Reservoir Simulation Symposium of the Society of Petroleum Engineers, New Orleans, La, USA, 1982, Also see SPE Journal, pp.14-26, 1985.

[5] K. Pruess and T. N. Narasimhan, "On fluid reserves and the production of superheated steam from fractured, vapordominated geothermal reservoirs," Journal of Geophysical Research, vol. 87, no. 11, pp. 9329-9339, 1982.

[6] J. W. Pritchett, "Efficient numerical simulation of nonequilibrium mass and heat transfer in fractured geothermal reservoirs," in Proceedings of the 22nd Stanford Workshop on Geothermal Reservoir Engineering, pp. 287-293, 1997.

[7] T. Ishido, Geothermal Reservoir Engineering, Japan Geothermal Energy Association, 2002.

[8] T. Ishido and J. W. Pritchett, "Characterization of fractured reservoirs using continuous self-potential measurements," in Proceedings of the 28th Stanford Workshop on Geothermal Reservoir Engineering, pp. 158-165, 2003.

[9] T. Ishido and J. W. Pritchett, "Numerical simulation of electrokinetic potentials associated with subsurface fluid flow," Journal of Geophysical Research B, vol. 104, no. 7, pp. 1524715259, 1999. 
[10] T. Ishido, Y. Nishi, and J. W. Pritchett, "Application of selfpotential measurements to geothermal reservoir engineering: characterization of fractured reservoirs," in Proceedings of the 35th Stanford Workshop on Geothermal Reservoir Engineering, 2010.

[11] S. Takakura, Y. Nishi, and T. Ishido, "Resistivity monitoring during an air injection experiment to fractured rocks," in Proceedings of the 123rd Meeting of SEGJ Extended Abstract, pp. 225-228, 2010.

[12] T. Negi, Y. Yoneda, and T. Senba, "Application of streaming potential method for detection of fractures in granite rock (part II)," in Proceedings of the 97th Meeting of SEGJ Extended Abstract, pp. 274-278, 1997.

[13] V. V. Bogoslovsky and A. A. Ogilvy, "Deformation of natural electric fields near drainage structures," Geophysical Prospecting, vol. 21, no. 4, pp. 716-723, 1973.

[14] T. Ishido, H. Mizutani, and K. Baba, "Streaming potential observations, using geothermal wells and in situ electrokinetic coupling coefficients under high temperature," Tectonophysics, vol. 91, no. 1-2, pp. 89-104, 1983.

[15] M. Darnet, A. Maineult, and G. Marquis, "On the origins of self-potential (SP) anomalies induced by water injections into geothermal reservoirs," Geophysical Research Letters, vol. 31, no. 19, article L19609, 2004.

[16] M. Darnet, A. Maineult, and G. Marquis, "On the origins of self-potential (SP) anomalies induced by water injections into geothermal reservoirs," Geophysical Research Letters, vol. 31, no. 19, article L19609, 2004.

[17] M.-Y. Chen, B. Raghuraman, I. Bryant, and M. Supp, "Streaming potential applications in oil fields," in Proceedings of SPE Annual Technical Conference and Exhibition, vol. 2, pp. 24-27, 2006, Paper SPE 102106.

[18] E. Rizzo, B. Suski, A. Revil, S. Straface, and S. Troisi, "Selfpotential signals associated with pumping tests experiments," Journal of Geophysical Research B, vol. 109, no. 10, article B10203, 2004.

[19] S. Straface, C. Fallico, S. Troisi, E. Rizzo, and A. Revil, "An inverse procedure to estimate transmissivity from heads and SP signals," Ground Water, vol. 45, no. 4, pp. 420-428, 2007.

[20] A. Maineult, E. Strobach, and J. Renner, "Self-potential signals induced by periodic pumping tests," Journal of Geophysical Research B, vol. 113, no. 1, Article ID B01203, 2008.

[21] M. Darnet, G. Marquis, and P. Sailhac, "Hydraulic stimulation of geothermal reservoirs: fluid flow, electric potential and microseismicity relationships," Geophysical Journal International, vol. 166, no. 1, pp. 438-444, 2006.

[22] T. Ishido and J. Muzutani, "Experimental and theoretical basis of electrokinetic phenomena in rock-water systems and its applications to geophysics," Journal of Geophysical Research, vol. 86, no. 3, pp. 1763-1775, 1981.

[23] T. Ishido and J. W. Pritchett, "Numerical simulation of electrokinetic potentials associated with subsurface fluid flow," in Proceedings of the 21st Stanford Workshop on Geothermal Reservoir Engineering, pp. 143-149, 1996.

[24] K. Yokoi, Y. Noguchi, H. Choh, and S. Hamabe, "Geophysical methods applied in underground investigation programs at the Kamaishi Mine," Busuri-Tansa, vol. 44, no. 6, pp. 350-361, 1991.

[25] Y. Nishi, T. Ishido, and T. Negi, "Field experiments to characterize hydrological properties of fractured rock using continuous borehole self-potential measurement," Transactions of Geothermal Resources Council, vol. 30, pp. 835-838, 2006.
[26] Y. Nishi, T. Ishido, and T. Negi, "Continuous borehole self-potential measurement-a new approach to characterize hydrological properties of fractured rock," Butsuri-Tansa, vol. 61, pp. 285-299, 2008.

[27] Y. Yoneda, H. Takahara, N. Nakamura, S. Akiyama, T. Moriya, and T. Negi, "Investigation of geological environment of deeper granitic rock at the Kamaishi Mine (H9)," Report PNC TJ 1380 98-002, Power Reactor and Nuclear Fuel Development, 1998.

[28] Y. Yoneda and T. Negi, "Geological surveys of fracture zone of deeper granitic rock at the Kamaishi Mine (H7)," PNC TJ 1380 96-001, Power Reactor and Nuclear Fuel Development, 1996.

[29] T. Ishido and N. Matsushima, "Streaming potential measured for an intact granite sample at temperatures to $200^{\circ} \mathrm{C}$," in Proceedings of the 32nd Stanford Workshop on Geothermal Reservoir Engineering, 2007.

[30] J. W. Pritchett and T. Ishido, "Numerical simulation of underground electrical signals caused by hydrofracturing operations," in Proceedings of the World Geothermal Congress, Bali, Indonesia, 2010.

[31] J. H. Saunders, M. D. Jackson, and C. C. Pain, "Fluid flow monitoring in oilfields using downhole measurements of electrokinetic potential," Geophysics, vol. 73, no. 5, pp. E165E180, 2008. 

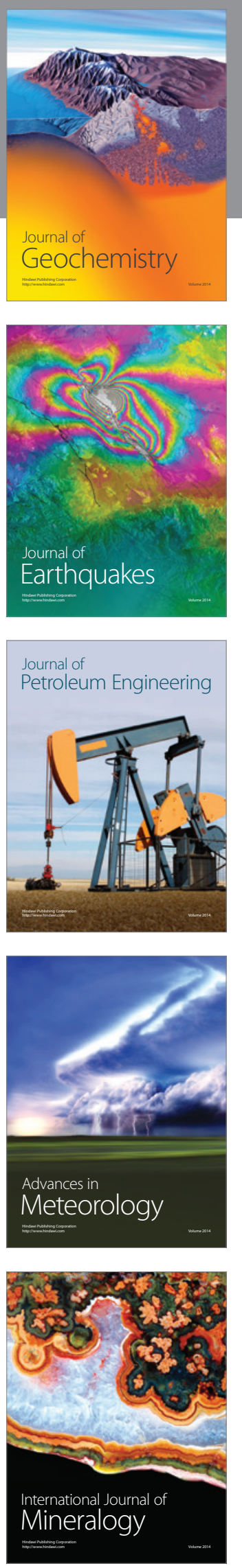
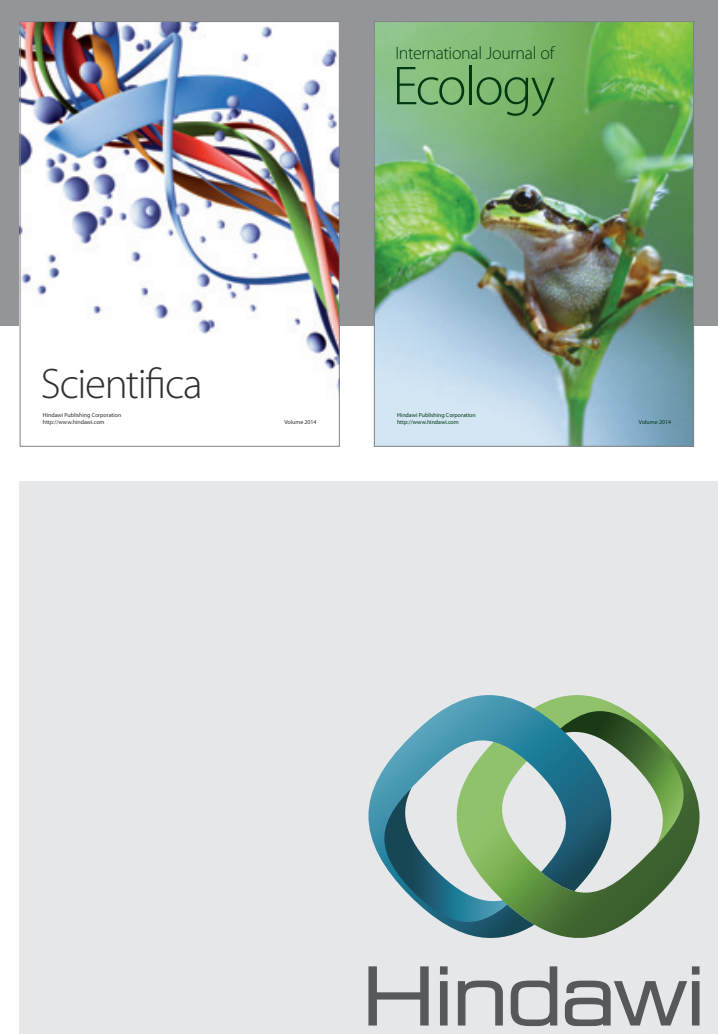

Submit your manuscripts at http://www.hindawi.com
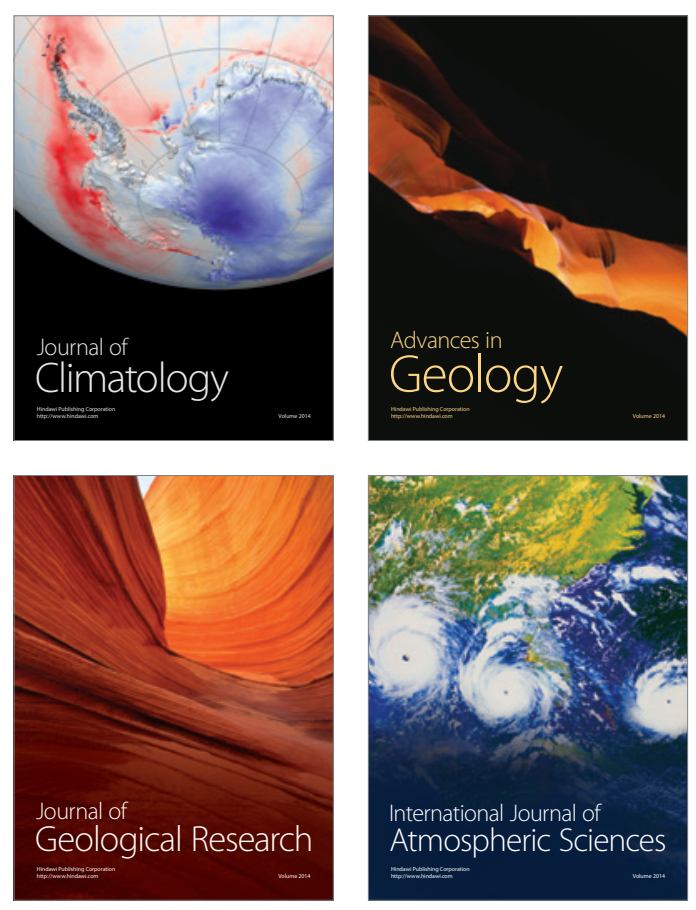
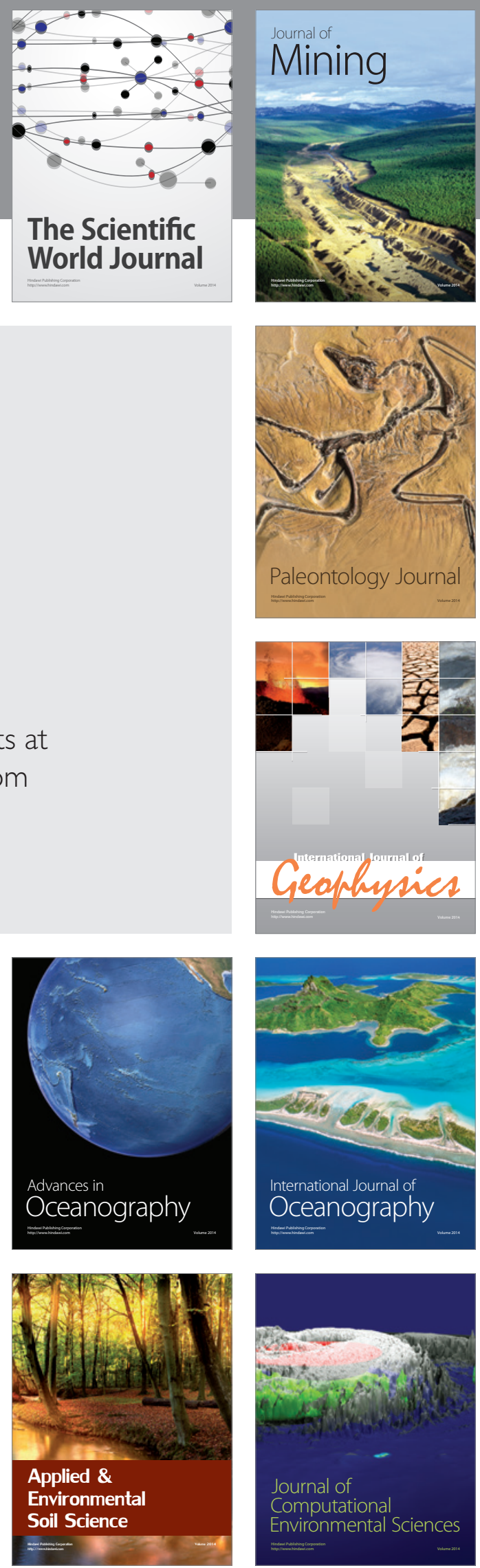